\title{
The Focused Ultrasound Myoma Outcome Study (FUMOS); a retrospective cohort study on long-term outcomes of MR-HIFU therapy
}

\author{
Inez M. Verpalen ${ }^{1,2}\left(\mathbb{D} \cdot\right.$ Jolien P. de Boer ${ }^{3} \cdot$ Marlot Linstra $^{4} \cdot$ Roelien L. I. Pol ${ }^{5} \cdot$ Ingrid M. Nijholt $^{1} \cdot$ Chrit T. W. Moonen $^{6}$ • \\ Lambertus W. Bartels $^{6,7} \cdot$ Arie Franx $^{8} \cdot$ Martijn F. Boomsma $^{1} \cdot$ Manon N. G. Braat ${ }^{2}$
}

Received: 8 August 2019 / Revised: 15 November 2019 / Accepted: 17 December 2019

(C) European Society of Radiology 2020

\begin{abstract}
Objectives Since 2004, uterine fibroids have been treated with MR-HIFU, but there are persevering doubts on long-term efficacy to date. In the Focused Ultrasound Myoma Outcome Study (FUMOS), we evaluated long-term outcomes after MR-HIFU therapy, primarily to assess the reintervention rate.

Methods Data was retrospectively collected from 123 patients treated with MR-HIFU at our hospital from 2010 to 2017. Followup duration and baseline (MRI) characteristics were retrieved from medical records. Treatment failures, adverse events, and the nonperfused volume percentage (NPV\%) were determined. Patients received a questionnaire about reinterventions, recovery time, satisfaction, and pregnancy outcomes. Restrictive treatment protocols were compared with unrestrictive (aiming for complete ablation) treatments. Subgroups were analyzed based on the achieved NPV $<50$ or $\geq 50 \%$.

Results Treatment failures occurred in $12.1 \%$ and the number of adverse events was $13.7 \%$. Implementation of an unrestrictive treatment protocol significantly $(p=0.006)$ increased the mean NPV\% from $37.4 \%$ [24.3-53.0] to 57.4\% [33.5-76.5]. At 63.5 \pm 29.0 months follow-up, the overall reintervention rate was 33.3\% $(n=87)$. All reinterventions were performed within 34 months follow-up, but within 21 months in the unrestrictive group. The reintervention rate significantly $(p=0.002)$ decreased from $48.8 \%$ in the restrictive group $(n=43$; follow-up $87.5 \pm 7.3$ months) to $18.2 \%$ in the unrestrictive group $(n=44$; follow-up $40.0 \pm$ 22.1 months). The median recovery time was 2.0 [1.0-7.0] days. Treatment satisfaction rate was $72.4 \%$ and $4 / 11$ women completed family planning after MR-HIFU.

Conclusions The unrestrictive treatment protocol significantly increased the NPV\%. Unrestrictive MR-HIFU treatments led to acceptable reintervention rates comparable to other reimbursed uterine-sparing treatments, and no reinterventions were reported beyond 21 months follow-up.
\end{abstract}

Electronic supplementary material The online version of this article (https://doi.org/10.1007/s00330-019-06641-7) contains supplementary material, which is available to authorized users.

Inez M. Verpalen

i.m.verpalen@isala.nl

1 Department of Radiology, Isala Hospital, Dokter van Heesweg 2, 8025 AB Zwolle, The Netherlands

2 Department of Radiology, University Medical Centre Utrecht, Utrecht, The Netherlands

3 Department of Gynaecology, Martini Hospital, Groningen, The Netherlands
4 Department of Gynaecology, Spaarne Gasthuis, Haarlem, The Netherlands

5 Department of Gynaecology, Isala Hospital, Zwolle, The Netherlands

6 Department of Molecular Imaging, University Medical Centre Utrecht, Utrecht, The Netherlands

7 Image Sciences Institute, Imaging Division, University Medical Centre Utrecht, Utrecht, The Netherlands

8 Department of Obstetrics and Gynaecology, Erasmus Medical Centre, Rotterdam, The Netherlands 


\section{Key Points}

- All reinterventions were performed within 34 months follow-up, but in the unrestrictive treatment protocol group, no reinterventions were reported beyond 21 months follow-up.

- The NPV\% was negatively associated with the risk of reintervention; thus, operators should aim for complete ablation during MR-guided HIFU therapy of uterine fibroids.

- Unrestrictive treatments have led to acceptable reintervention rates after MR-guided HIFU therapy compared to other reimbursed uterine-sparing treatments.

Keywords Uterine fibroids · MR-guided interventional procedures $\cdot$ High-intensity-focused ultrasound ablation

$\begin{array}{ll}\text { Abbreviations } \\ \text { CTCAE } & \text { Common Terminology Criteria for Adverse } \\ & \text { Events } \\ \text { DISC } & \text { Direct skin cooling } \\ \text { MREC } & \text { Medical Research Ethics Committee Board } \\ \text { MR-HIFU } & \text { Magnetic resonance image-guided high- } \\ & \text { intensity-focused ultrasound } \\ \text { MRI } & \text { Magnetic resonance imaging } \\ \text { NPV } & \text { Nonperfused volume } \\ \text { RFA } & \text { Radiofrequency ablation } \\ \text { UAE } & \text { Uterine artery embolization } \\ \text { UMCU } & \text { University Medical Centre Utrecht }\end{array}$

\section{Introduction}

Uterine fibroids have a high lifetime prevalence varying between 70 and $80 \%$ [1]. In $25 \%$ of women, uterine fibroids cause clinically significant symptoms. Symptoms can be classified into three categories: abnormal menstrual bleeding, bulk-related symptoms, and reproductive dysfunction [2, 3]. To date, hysterectomy is still the most commonly performed intervention for uterine fibroids. Although effective in reducing symptoms, hysterectomy is associated with a risk of postoperative complications and requires several weeks of recovery $[4,5]$. Myomectomy is considered the best therapeutic choice for women desiring pregnancy [6]. Less invasive treatment options are available including uterine artery embolization (UAE) and radiofrequency ablation (RFA). Magnetic resonance image-guided high-intensity-focused ultrasound (MRHIFU) is a completely noninvasive therapy which combines high-intensity-focused ultrasound for tissue heating with realtime MRI and MRI-based temperature monitoring for therapy guidance [7]. A pretreatment MRI is necessary to determine patient suitability for MR-HIFU [8]. Importantly, not all fibroids are suitable for each treatment strategy, but these less invasive approaches have the obvious advantage of uterine preservation and allow future childbearing. Other benefits include less pain posttreatment, less complications, shorter hospital stay, and faster recovery $[9,10]$. However, these therapies have been associated with a higher fibroid recurrence risk. A systematic review reported a reintervention rate after 3 years of $7.4 \%$ for UAE, $10.4 \%$ for RFA, and $34.7 \%$ for MR-HIFU compared to $1.2 \%$ for myomectomy [11]. Although that review included MR-HIFU studies using outdated treatment protocols and older devices, there are persevering doubts on the long-term efficacy of MR-HIFU. An important predictor of MR-HIFU treatment success is the ablated fibroid volume (Fig. 1), e.g., the nonperfused volume (NPV). A higher NPV\% results in greater fibroid shrinkage, improved relief of symptoms, and lower retreatment rates [12-14]. Technological improvements and treatment protocol modifications are nowadays leading to increased NPV\% $[15,16]$. Importantly, data beyond 24 months follow-up of MR-HIFU treatments with protocols aiming for complete fibroid ablation is scarce [14].

\section{Objectives}

This study was conducted for the evaluation of long-term outcomes after MR-HIFU therapy of uterine fibroids, primarily to assess the reintervention rate. Secondary outcomes included safety, treatment failures, NPV\%, treatment satisfaction, and reproductive outcomes.

\section{Materials and methods}

\section{Study design}

In this retrospective study, women treated with MR-HIFU from April 2010 to December 2017 at the University Medical Centre Utrecht (UMCU) in the Netherlands were included. Data from part of this patient population was used in previous studies [17-20]. The research protocol (reference number 17-892) was examined by the Medical Research Ethics Committee Board (MREC) UMCU and confirmed on the 18 th of January 2018 that the Medical Research Involving Human Subjects act (WMO) does not apply to our study. Data was retrospectively retrieved from medical records. Additional follow-up data was collected using a questionnaire (see ESM Appendix 1) by mail. All patients signed informed consent before the initial MR-HIFU treatment and a renewed informed consent before filling in the questionnaire. 


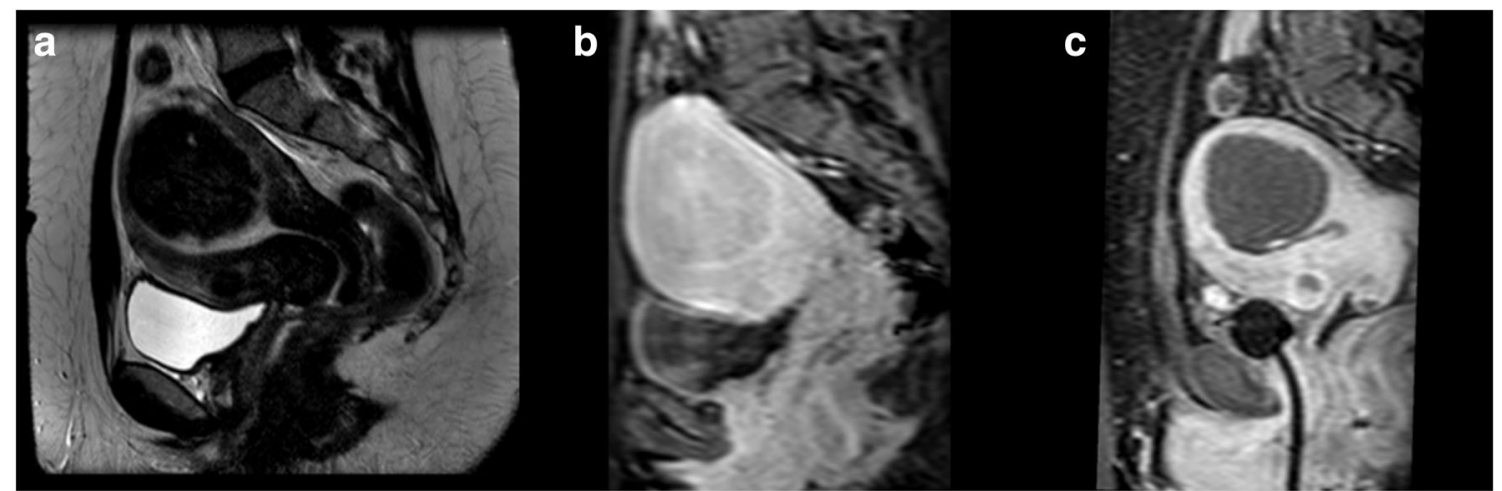

Fig. 1 Pretreatment and posttreatment images: a T2-weighted image from the screening MRI, (b) gadolinium-enhanced T1-weighted image from the screening MRI, and (c) the same sequence immediately post-MR-HIFU treatment

\section{MR imaging and MR-HIFU treatment}

Two radiologists carried out all MR-HIFU procedures, without prior MR-HIFU experience. The first radiologist consecutively treated the first 78 patients and the second radiologist consecutively treated the last 45 patients. Patients were treated on a clinical MR-HIFU system (Sonalleve, Profound Medical Inc.) integrated into a 1.5-T MRI (Achieva, Philips Healthcare) [16]. During the study period, an upgrade was installed (Sonalleve V1 to V2) including a direct skin cooling (DISC) system. Furthermore, two different MR-HIFU treatment protocols were used. Therefore, the cohort was subdivided into two subgroups: restrictive treatment protocol versus unrestrictive treatment protocol. During the first treatments [18, 19], restrictive guidelines were followed for safety measures: patient immobilization time was limited to 3 hours, no sensitive structures (i.e., bone, bowel, scar tissue, clips, bladder with catheter, or nerves) in the near field beam path, far field safety margin $(30 \mathrm{~mm})$ to the spine (or other sensitive structures), and a cell safety margin $(10 \mathrm{~mm})$ from the treatment cell to the uterine serosa. The unrestrictive treatment protocol allowed for complete fibroid ablation without time limit or safety margins (the sonication spot was determined by the operator with careful assessment of neighboring sensitive structures).

\section{Data collection}

Information from medical records was retrieved about (a) patient characteristics, (b) pretreatment MR parameters, (c) occurrence of (serious) adverse events; (d) treatment failures, and (e) NPV\% immediately post-MR-HIFU treatment. Follow-up duration was calculated from the initial MR-HIFU treatment till 04 August 2018. On the pretreatment MRI, the following measurements were performed: the thickness of the abdominal subcutaneous fat layer, the number of fibroids, the maximum diameter, and the total fibroid volume by semiautomatic segmentation in the tumor tracking function of IntelliSpace Portal (ISP) software (Philips Healthcare) with review and manual correction of the segmentation result by an expert. MRI fibroid characteristics consisted of the presence of contrast enhancement on T1-weighted images and the fibroid's signal intensity on the T2-weighted images to determine Funaki classification [21]. Based on their T2 signal intensity, fibroids can be classified into three Funaki subtypes (Fig. 2). Funaki types 1 and 2 respond well to MR-HIFU therapy, while treatment of Funaki type 3 fibroids is not recommended [22]. Treatment failures were defined as treatments canceled due to bowel interposition, device malfunction, patient discomfort, or inadequate heating of the uterine fibroid. MRHIFU treatment failures were not included in further analysis. Adverse events were identified and graded according to the Common Terminology Criteria for Adverse Events (CTCAE) [23]. On the posttreatment MR images, the NPV was volumetrically measured in ISP and the NPV\% was calculated by the following formula: (NPV/fibroid volume) $\times 100 \%$. The need for additional treatment was based on clinical evaluation. The radiological post-HIFU evaluation was not used to propose additional treatments. Followup data on the need for additional treatment during followup, recovery time in days, patient treatment satisfaction, and pregnancy outcomes were collected using a questionnaire. A reintervention was defined as second MR-HIFU treatment, embolization, myomectomy, or hysterectomy. The continued use of medication was not considered a reintervention. Repeat MR-HIFU treatments for other fibroids or large fibroids scheduled in two tempi were not considered reinterventions. A subanalysis for the reintervention rate was performed based on the achieved NPV $<50$ or $\geq 50 \%$, and this cutoff point is in concordance with previous publications $[12,17,24,25]$. Until 2013, a wish for future pregnancy was an exclusion criterion. Despite this, all patients were asked if they had a desire for future pregnancy prior to the MR-HIFU treatment and whether they conceived during follow-up. 


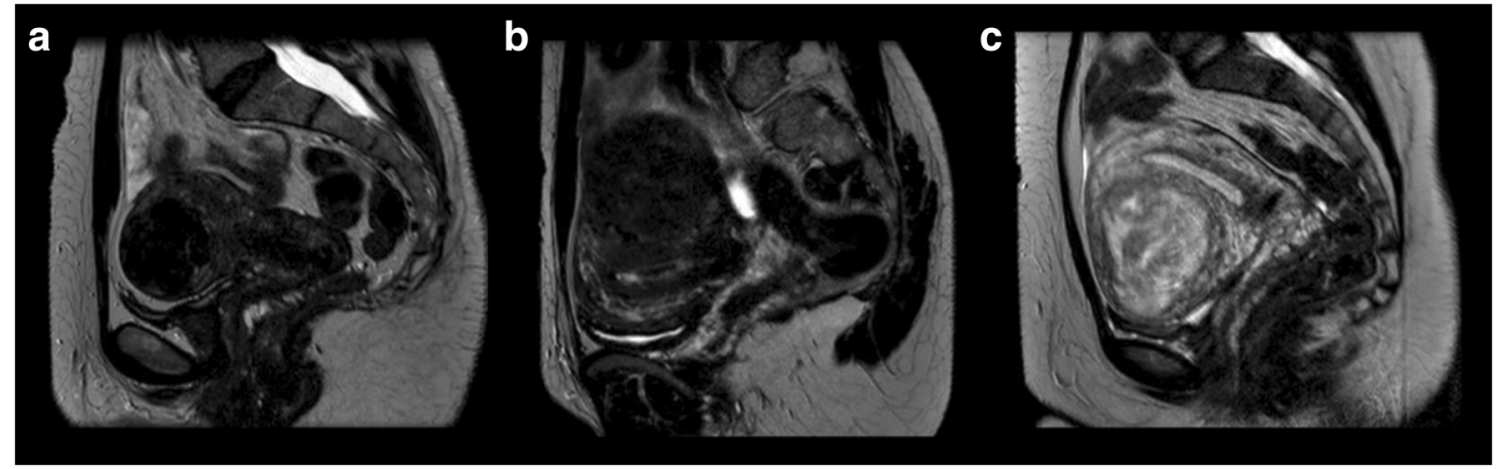

Fig. 2 Funaki classification: a Funaki I fibroid (signal intensity lower than myometrium and muscle), (b) Funaki II fibroid (signal intensity lower than myometrium, but higher than muscle), and (c) Funaki III fibroid (signal intensity higher than muscle and myometrium)

\section{Statistics}

For statistical analysis, IBM SPSS Statistics 25 was used. The level of statistical significance was set at $p<0.05$. A Mann-Whitney $U$ test was used to calculate significant difference of NPV\% between treatment protocols and reintervention groups and to compare follow-up duration between subgroups. Chi-square test was used to calculate significant difference of treatment failures and reintervention rate between Funaki types, treatment protocols, and NPV subgroups. A Cox regression was used to evaluate the relationship between undergoing a reintervention (outcome) and the NPV\% achieved immediately after treatment (predictor) as well as the relationship between a reintervention and the treatment protocol used. Cox regression was performed and corrected for any possible confounding effects such as NPV\% and treatment protocol. The probability of undergoing a reintervention as a function of time was analyzed by a Kaplan-Meier curve. The NPV\% was compared between patients lost to follow-up and patients who completed the follow-up. We stratified outcomes by system (V1/V2), treating radiologist, treatment protocol, NPV\%, followup duration, and Funaki type.

\section{Results}

One hundred and twenty-three patients were treated with MR-HIFU between April 2010 and December 2017. The flowchart of the selection process is shown in Fig. 3. Eleven patients were excluded from the analysis. A total of 124 MR-HIFU treatments were performed on 112 patients. Twelve patients were treated in two tempi due to (a) treatment volume of the fibroid which was not treatable in a single session, (b) the treatment of multiple fibroids, and (c) the occurrence of technical failure during the first treatment.

\section{Procedure-related outcomes}

Overall, 17 adverse events occurred (13.7\%) in 124 treatments. The reported adverse events were skin redness, firstand second-degree skin burns, abdominal pain, malaise, vaginal discharge or bleeding, hematuria, cystitis, and neuropraxia (Table 1). Only mild (grade $1 ; n=13$ ) and moderate (grade $2 ; n=4$ ) adverse events were reported. Stratification by system, treatment protocol, or treating radiologist showed no significant differences in the total number of

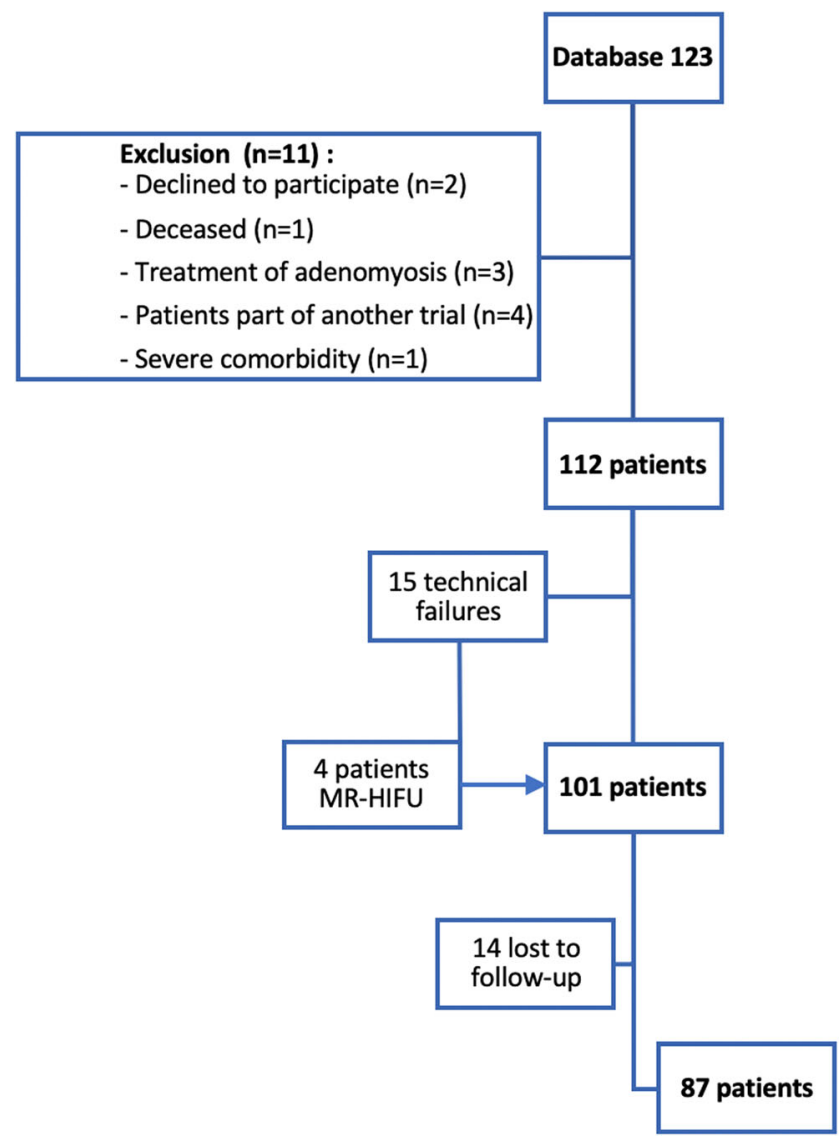

Fig. 3 Flowchart of participants 
Table 1 Specification of adverse events $(n=17)$ during 124 treatments on the left and specification of causes of treatment failure $(n=15)$ on the right

\begin{tabular}{lclr}
\hline Adverse events & $n(\%)$ & Treatment failures & $n(\%)$ \\
\hline Total & $17(13.7)$ & Total & $15(12.1)$ \\
Skin redness & $3(2.4)$ & Inadequate heating & $4(3.2)$ \\
Skin burns $^{\mathrm{a}}$ & $4(3.2)$ & Treatment of a Funaki 3 fibroid & $3(2.4)$ \\
Cystitis $_{\text {Abdominal pain }}{ }^{\mathrm{b}}$ & $1(0.8)$ & Physical discomfort, movement, and/or pain & $3(2.4)$ \\
Malaise & $3(2.4)$ & Interposition of intestine & $2(1.6)$ \\
Vaginal discharge or bleeding $^{\mathrm{b}}$ & $1(0.8)$ & Failure of the technology & \\
Hematuria $_{\text {Neuropraxia }}^{\mathrm{c}}$ & $3(2.4)$ & Vasovagal episode during treatment & \\
\hline
\end{tabular}

${ }^{a}$ Only first- and second-degree skin burns were reported

${ }^{\mathrm{b}}$ One woman reported abdominal and pain vaginal bleeding

${ }^{\mathrm{c}}$ Neuropraxia of L5 (caused by nontarget heating) and neuropraxia of the brachial plexus (due to positioning difficulties in the bore) were reported

adverse events. However, all skin burns were reported in treatments performed with the V1.

Treatment failures occurred in $12.1 \%$ (15/124) of the MRHIFU treatments (Table 1). Four patients underwent a second MR-HIFU treatment. The other patients $(n=11)$ chose not to undergo a second MR-HIFU treatment and were excluded from further analysis. Stratification by system or treating radiologist showed no significant differences in treatment failures.

The mean NPV\% of the remaining 101 patients was $48.4 \pm$ $25.0 \%(2.1-100.0)$. The median NPV\% was significantly different between the restrictive (37.4\% [24.3-53.0], $n=47)$ and unrestrictive (57.4\% [33.5-76.5], $n=54)$ treatment protocol, $p=0.006$. The median NPV\% of the patients that returned the questionnaire was lower (41.0 [28.3-66.1], $n=87$ ) compared to the patients who did not return the questionnaire $(65.3$ [38.3-77.7], $n=14$ ).

\section{Clinical outcomes}

In total, 87/101 patients returned the questionnaires (Fig. 3). Baseline characteristics are shown in Table 2. The mean follow-up duration was $63.5 \pm 29.0$ months (range 8 100 months).

Twenty-nine of the 87 patients (33.3\%) required additional treatment during the follow-up period due to fibroid-related symptoms (Table 3 ), consisting of 6 second MR-HIFU therapies $(21 \%), 4 \operatorname{UAE}(14 \%), 2$ myomectomies $(7 \%)$, and 17 hysterectomies $(58 \%)$.

Longer follow-up was associated with a higher risk for a reintervention (Fig. 4). All reinterventions were performed within 34 months of the initial MR-HIFU treatment.

We found a significant difference $(p=0.012)$ in NPV\% between patients who underwent a reintervention $(n=29)$ and patients with no reintervention $(n=58)$ with a median NPV\% of $35.7 \%$ [26.9-44.9] and 49.7\% [30.2-73.3], respectively. Furthermore, NPV\% was negatively associated with the risk of reintervention (HR 0.977 (95\% CI 0.961$0.994), p=0.009$ ). Analysis showed no confounding effect of treatment protocol regarding NPV\% prediction of outcome.

Comparison of the two treatment protocols $(p=0.002)$ showed that more patients treated with the restrictive protocol (21/43) needed additional treatment than patients treated with the unrestrictive protocol $(8 / 44)-48.8$ versus $18.2 \%$ (Table 3). Cox regression confirmed this: the unrestrictive treatment protocol lowers the risk of reintervention (HR 0.275 (95\% CI 0.111-0.681), $p=0.005$ ). The follow-up of the patients treated with an unrestricted protocol was also shorter $(40.0 \pm 22.1$ vs. $87.5 \pm 7.3$ months, $p<0.001)$ However, no reintervention was reported in this subgroup beyond 21 months follow-up.

A subanalysis was performed based on the achieved NPV\%. In 36 of the 87 patients (41.4\%), a NPV $\geq 50 \%$ was achieved. The reintervention percentage of these 36 patients was $16.7 \%(6 / 36)$, whereas 23 patients out of 51 patients with a NPV $<50 \%(45.1 \% ; p=0.021)$ required additional intervention during follow-up (Table 3 ). The mean follow-up duration was not significantly different for these two groups $(p=$ $0.281)-58.7 \pm 30.2(9-99)$ months and $66.8 \pm 28.0(8-100)$ months, respectively.

Stratification of the Funaki types led to a reintervention rate of $100 \%$ in Funaki type 3 fibroids (2/2), 39.3\% for type 2 (24/ $61)$, and $12.5 \%$ for type $1(3 / 24)$. The reintervention rate between Funaki 2 and Funaki 3 fibroids was not significantly different $(p=0.086)$, but was significant between Funaki 1 and Funaki 2 fibroids $(p=0.017)$. Funaki 3 fibroid treatments had a failure rate of $60 \%(6 / 10)$, compared to $10.7 \%(8 / 75)$ for Funaki 2 and 3.7\% (1/27) for Funaki 1 fibroids. This treatment failure rate was significantly different for Funaki 2 and 3 fibroids $(p=0.001)$, but not for Funaki 1 and 2 fibroids $(p=0.274)$. 
The median recovery time before patients returned to work or their normal activities was 2.0 [1.0-7.0] (0-60) days.

Fifty-eight out of 87 patients $(66.7 \%)$ reported about their treatment satisfaction. The other $29(33.3 \%)$ had difficulties remembering because too much time had passed since the treatment. Of those 58 patients, 42 patients $(72.4 \%)$ were satisfied with the treatment procedure at the hospital and 51/58 patients $(87.9 \%)$ would recommend this treatment to other women.

In total, 63 patients answered the questions regarding pregnancy of which 11 still had a desire for future pregnancy when they underwent MR-HIFU therapy. Four women (36.4\%) conceived, resulting in nine pregnancies two of which resulted in early pregnancy losses and seven in livebirths (six at term and one preterm). Mode of delivery was a Cesarean section in three cases and two vaginal deliveries. Reported complications during pregnancy were fibroid's necrosis, obstruction of labor $(n=1)$, and postpartum hemorrhage $(n=2)$.

\section{Discussion}

To the best of our knowledge, this retrospective study has the longest follow-up after MR-HIFU treatment of uterine fibroids reported to date. Previous publications all had a follow-up of 2 years or shorter [26, 27]. Only Mohr-Sasson

Table 2 Baseline characteristics of all patients $(n=87)$

\begin{tabular}{ll}
\hline Baseline characteristics* & \\
\hline Number of patients & 87 \\
Age (years) & $44.6 \pm 4.7(30.8-54.1)$ \\
& $44.5[41.2-48.2]$ \\
Duration of follow-up (months) & $63.5 \pm 29.0(8-100)$ \\
& $74.0[37.0-88.0]$ \\
Number of fibroids & \\
1 & $39(44.8)$ \\
2 & $13(14.9)$ \\
3 & $8(9.2)$ \\
4 & $10(11.5)$ \\
$\geq 5$ & $17(19.5)$ \\
Targeted fibroid diameter $\left(\mathrm{cm}^{*}\right)$ & $9.3 \pm 3.1(3-18.9)$ \\
Targeted fibroid volume $\left(\mathrm{cm}^{3}\right)$ & $9.1[7.0-11.5]$ \\
Targeted fibroid Funaki intensity & $347.3 \pm 260.8(7.4-1490.3)$ \\
1 & $305.0[158.0-505.2]$ \\
2 & \\
3 & $24(27.6)$ \\
Subcutaneous fat layer $\left(\mathrm{cm}^{3}\right)$ & $61(70.1)$ \\
& $2(2.3)$ \\
& $1.2 \pm 0.6(0.1-3.1)$ \\
& $1.0[0.8-1.6]$ \\
\hline
\end{tabular}

*Data presented as $n, n(\%)$, or mean $\pm \mathrm{SD}($ range) and median [Q1-Q3]
Table 3 Clinical outcomes at the end of follow-up

Follow-up outcomes*

\begin{tabular}{lc}
\hline Duration of follow-up (months) $(n=87)$ & $63.5 \pm 29.0(8-100)$ \\
& $74.0[37.0-88.0]$ \\
Recovery time (days) $(n=87)$ & $5.8 \pm 9.7(0-60)$ \\
& $2.0[1.0-7.0]$ \\
Treatment satisfaction rate $(n=58 / 87)$ & \\
Satisfied & $42 / 58(72.4)$ \\
Unsatisfied & $16 / 58(28.6)$ \\
Reinterventions & $29 / 87(33.3)$ \\
Hysterectomy & $17 / 29(58.0)$ \\
Myomectomy & $2 / 29(7.0)$ \\
Uterine artery embolization & $4 / 29(14.0)$ \\
Repeat MR-HIFU & $6 / 29(21.0)$ \\
Reintervention subgroup analysis & \\
Restrictive protocol group $(n=43)^{\mathrm{a}}$ & $21 / 43(48.8)$ \\
Unrestrictive protocol group $(n=44)^{\mathrm{a}}$ & $8 / 44(18.2)$ \\
NPV $<50 \%(n=51)^{\mathrm{c}}$ & $23 / 51(45.1)$ \\
NPV $\geq 50 \%(n=36)^{\mathrm{c}}$ & $6 / 36(16.7)$ \\
Funaki $1(n=24)$ & $3 / 24(12.5)$ \\
Funaki $2(n=61)$ & $24 / 61(39.3)$ \\
Funaki $3(n=2)$ & $2 / 2(100)$
\end{tabular}

*Data presented as $n, n(\%)$, or mean $\pm \mathrm{SD}$ (range) and median [Q1-Q3]

${ }^{\mathrm{a}}$ The follow-up of patients treated with an unrestricted protocol was significantly shorter $-40.0 \pm 22.1$ versus $87.5 \pm 7.3$ months (median 41.0 [18.3-62.0] vs. 88.0 [82.0-94.0] months)

${ }^{\mathrm{b}}$ Nonperfused volume percentage

${ }^{\mathrm{c}}$ The follow-up between the NPV subgroups was not significantly different $-58.7 \pm 30.2$ versus $66.8 \pm 28.0$ months (median 62.5 [29.5-89.8] vs. $79.0[47.0-87.0]$ months

et al had an average follow-up of 36.5 months [28]. Our mean follow-up of 63.5 months exceeds that study.

Similar to our study, longer follow-up is associated with a higher risk for a reintervention [29, 30]. However, all reinterventions were performed within 34 months follow-up and no reinterventions were reported beyond 21 months follow-up in the unrestrictive treatment protocol group. Although this might be partially explained by women becoming postmenopausal, it is an important finding for future study designs.

Our reintervention rate after MR-HIFU was 33.3\%, which is relatively high, but our studies included treatment with both restricted and unrestricted protocols, and it can be expected that the reintervention rate in this study is higher than studies only including treatment protocols aiming for full ablation, as illustrated by our nonrestrictive treatments which decreased the reintervention rate to $18.2 \%$.

The probability of requiring reintervention decreases as the NPV\% increases [13]. Our reintervention rate was lower when the achieved NPV\% was $\geq 50 \%$ (16.7 vs. $45.1 \%$ ), and implementation of an unrestricted treatment protocol led to a higher 
Fig. 4 Probability of undergoing reintervention over time (months), stratified by treatment protocol showing a significant difference $(p=0.005)$

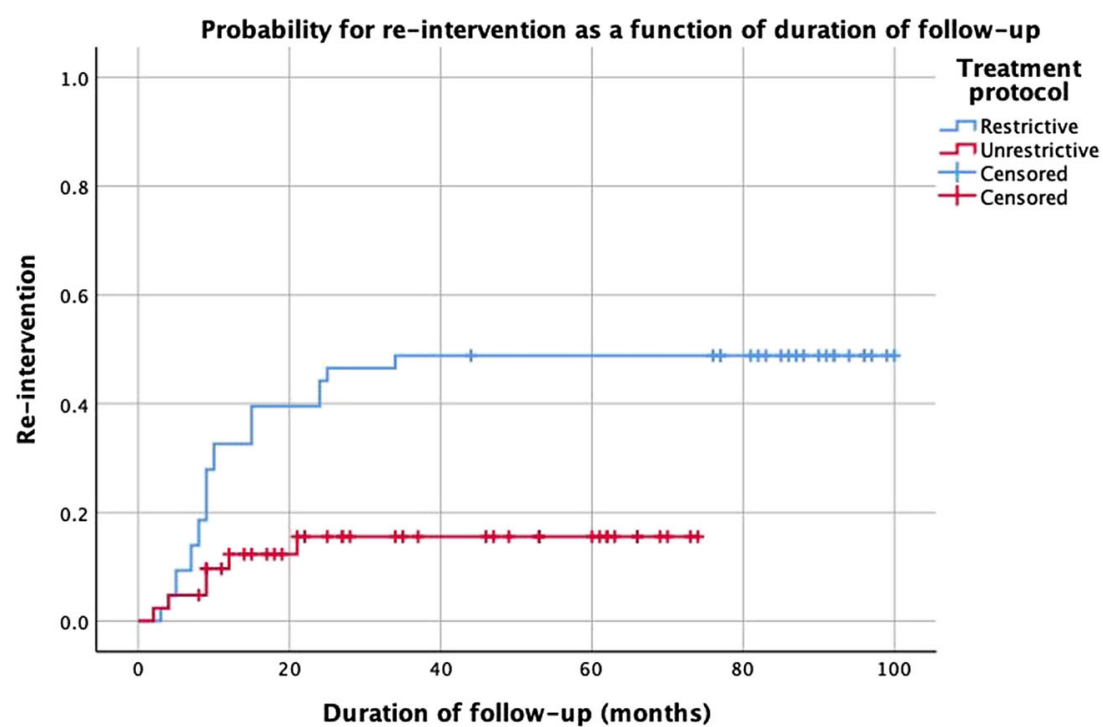

mean NPV\% [16]. Therefore, operators nowadays should aim for complete ablation to reduce the reintervention risk.

As outlined above, none of the earlier MR-HIFU studies had a comparable follow-up duration, but 5-year outcomes after ultrasound-guided HIFU treatments have been reported previously. Sandberg et al compared long-term reintervention rates after several uterine-sparing treatments of uterine fibroids [11]. At 60 months, the reintervention rate was $12.2 \%$ for myomectomy, $14.4 \%$ for UAE, and $53.9 \%$ after MRHIFU which is higher than our reintervention rate of $33.3 \%$.

Two studies compared MR-HIFU to UAE [17, 31], and they reported lower reintervention rates and greater improvement in symptoms after UAE. However, the mean NPV\% in both trials was below 50\% [32]. More recent studies reported lower retreatment rates: $12.7 \%$ after MR-HIFU at 19.4 months (comparable to UAE) [13, 28]. The latter study compared long-term outcomes of MR-HIFU to laparoscopic myomectomy and found no significant differences in symptom reduction, quality of life, or reintervention rates.

Importantly, the average recovery time after MR-HIFU in our study was $5.8 \pm 9.7$ days, compared to $22.1 \pm 12.3$ days for myomectomy and $11.9 \pm 5.9$ days for UAE [33]. This implies both reduced hospital stay as absenteeism, which decreases both the economic and social burden of uterine fibroids. Further large controlled trials, comparing the presently available different treatments for uterine fibroids and utilizing the newest equipment and treatment protocols, are needed to confirm these findings.

The number of patients desiring pregnancy in this study was too small to determine the pregnancy rate, because a desire for future childbearing was an exclusion criterion until 2013 [18]. However, similar to previous cohort studies, the pregnancy outcomes after MR-HIFU treatment in this study were reassuring [34], contrary to UAE, which can compromise ovarian reserve, and thereby fertility, as suggested by the significantly decrease in anti-Mullerian hormone levels compared to HIFU in the FIRSTT study [31]. Moreover, MRHIFU also has the benefit of no waiting period before attempting to conceive. Therefore, MR-HIFU might be a promising treatment for women desiring pregnancy, but the effect of MR-HIFU on fertility should be examined in more detail.

Our study demonstrated that MR-HIFU treatment was safe because the number of adverse events was acceptable. However, the treatment failure rate in this study was relatively high (12.1\%). This could be partially explained by two learning curves of both radiologists without prior MR-HIFU ablation experience [35]. Additionally, bowel-interference mitigation techniques were not implemented, which may have resulted in more treatment failures or low NPV\% due to interposition of small bowel loops at the day of the MR-HIFU treatment [36]. Furthermore, none of the patients with Funaki type 3 fibroids had successful MR-HIFU treatment, underlining that these fibroids are difficult to treat and that better fibroid selection could further decrease the reintervention rate [22].

Limitations of this study were related to the design of a nonrandomized, retrospective cohort study. Recall bias is a limitation as with all survey studies. In addition, the risk at a selection bias was high, because treatment choice was based on patient's preference. Furthermore, two learning curves of the treating radiologists may have complicated the evaluation of our treatment results, but we expected that the treatment protocol subgroups were equally influenced because the second learning curve started during the unrestrictive treatments. Additionally, the treatment of Funaki type 3 fibroids affected our reintervention analyses. Another study limitation is the difference in follow-up duration between the treatment protocols. Moreover, the mean NPV\% was below 50\% which is lower than achieved in more recently published studies [13, 
$28,37]$. This can be explained by operator experience, restrictive treatment guidelines, and technological improvements. For example, the greater maximum depth of the Sonalleve $\mathrm{V} 2$ and the reduced cooling times with DISC both result in larger NPV\% (and subsequently lower reintervention rates). Lastly, the assessment of NPV\% was done immediately following MR-HIFU when apoptosis effects have not been completed yet [38-40]. Therefore, the NPV\% is systematically underestimated.

Specific patient groups may benefit from MR-HIFU such as women with a desire to conceive or as a bridge to menopause in older women. The best treatment option for uterine fibroids is influenced by a woman's symptoms, age, pregnancy wish, fibroid location, and patient's preference [41]. Besides, final therapy is also influenced by fibroid characteristics since not all fibroids are suitable for each treatment strategy. This illustrates the importance of personalized healthcare for women with uterine fibroids.

\section{Conclusion}

The mean follow-up duration was 63.5 months, but all reinterventions were performed within 34 months. Importantly, no reinterventions were reported beyond 21 months in the unrestrictive treatment protocol group. The NPV\% was negatively associated with the risk of reintervention; thus, operators should aim for complete fibroid ablation. Unrestrictive MR-HIFU treatments have led to acceptable reintervention rates compared to other reimbursed uterine-sparing treatments.

Authors' contributions IV made substantial contribution to the design, interpretation of data, and writing of the manuscript. JP made substantial contribution to the study design and obtaining ethical approval. ML had a major contribution in the collection of data and analyzing of the data. RP made a substantial contribution to the interpretation of the data and the writing of the manuscript. IN was involved in the analysis and the interpretation of data and revised the manuscript. CM had substantial contribution to the design of the study and revised the manuscript. LB and AF revised the manuscript critically for important intellectual content. MFB was involved in the interpretation of data and revised the manuscript. MNGB was responsible for the design of the study, facilitated the collection of data and interpretation of the data, and revised the manuscript. All authors read and approved the final manuscript.

Funding information No grant or financial support was used for this research project. No author had any financial interest in the subject matter discussed in the submitted manuscript. All the authors state that this study complies with the Declaration of Helsinki.

\section{Compliance with ethical standards}

Guarantor The scientific guarantor of this publication is M.F. Boomsma, M.D., Ph.D.
Conflict of interest The authors declare that they have no conflict of interest.

Statistics and biometry One of the authors has significant statistical expertise: I.M. Nijholt, Ph.D. No complex statistical methods were necessary for this paper.

Informed consent Written informed consent was obtained from all subjects (patients) in this study.

Ethical approval Institutional Review Board approval was obtained.

Study subjects or cohorts overlap The short- and mid-term results of some study subjects have been previously reported in the following: - Ikink ME, Nijenhuis RJ, Verkooijen HM et al (2014) Volumetric MRguided high-intensity focused ultrasound versus uterine artery embolisation for treatment of symptomatic uterine fibroids: comparison of symptom improvement and reintervention rates. Eur Radiol 24:2649-2657. https://doi.org/10.1007/s00330-014-3295-6

- Ikink ME, Voogt MJ, Verkooijen HM et al (2013) Mid-term clinical efficacy of a volumetric magnetic resonance-guided high-intensity focused ultrasound technique for treatment of symptomatic uterine fibroids. Eur Radiol 23:3054-61. https://doi.org/10.1007/s00330-013-2915-x

- Voogt MJ, Trillaud H, Kim YS et al (2012) Volumetric feedback ablation of uterine fibroids using magnetic resonance-guided high intensity focused ultrasound therapy. Eur Radiol 22:411-417. https://doi.org/10. 1007/s00330-011-2262-8

- Ikink ME, van Breugel JMM, Schubert G et al (2015) Volumetric MRguided high-intensity focused ultrasound with direct skin cooling for the treatment of symptomatic uterine fibroids: proof-of-concept study. BioMed Research International 2015:1-10. https://doi.org/10.1155/ 2015/684250

\section{Methodology}

- Retrospective

- Case-control study

- Performed at one institution

\section{References}

1. Baird DD, Dunson DB, Hill MC, Cousins D, Schectman JM (2003) High cumulative incidence of uterine leiomyoma in black and white women: ultrasound evidence. Am J Obstet Gynecol 188(1):100 107

2. Stewart EA (2015) Clinical practice. Uterine fibroids. N Engl J Med 372:1646-1655. https://doi.org/10.1056/NEJMcp1411029

3. Stewart EAM, Nicholson WKM, Bradley LM, Borah BJP (2013) The burden of uterine fibroids for African-American women: results of a national survey. J Womens Health (Larchmt) 22:807-816. https://doi.org/10.1089/jwh.2013.4334

4. Spies JB, Bradley LD, Guido R, Maxwell GL, Levine BA, Coyne K (2010) Outcomes from leiomyoma therapies: comparison with normal controls. Obstet Gynecol 116:641-652. https://doi.org/10. 1097/AOG.0b013e3181ed36b3

5. Spilsbury K, Semmens J, Hammond I, Bulsara M (2009) Morbidity outcomes of 78577 hysterectomies for benign reasons over 23 years. BJOG 116:734-735. https://doi.org/10.1111/j.1471-0528. 2008.02094.x

6. Kotani Y, Tobiume T, Fujishima R et al (2018) Recurrence of uterine myoma after myomectomy: open myomectomy versus laparoscopic myomectomy. J Obstet Gynaecol Res 44:298-302. https:// doi.org/10.1111/jog.13519 
7. Hynynen K (2011) MRIgHIFU: a tool for image-guided therapeutics. J Magn Reson Imaging 34:482-493. https://doi.org/10.1002/ jmri.22649

8. Kim YS, Lee JW, Choi CH et al (2016) Uterine fibroids: correlation of T2 signal intensity with semiquantitative perfusion MR parameters in patients screened for MR-guided high-intensity focused ultrasound ablation. Radiology 278:925-935. https://doi.org/10. 1148/radiol.2015150608

9. Silberzweig JE, Powell DK, Matsumoto AH, Spies JB (2016) Management of uterine fibroids: a focus on uterine-sparing interventional techniques. Radiology 280:675-692. https://doi.org/10. 1148/radiol.2016141693

10. Ravina JH, Herbreteau D, Ciraru-Vigneron N et al (1995) Arterial embolisation to treat uterine myomata. Lancet (London, England) 346:671-672

11. Sandberg EM, Tummers FHMP, Cohen SL, van den Haak L, Dekkers OM, Jansen FW (2018) Reintervention risk and quality of life outcomes after uterine-sparing interventions for fibroids: a systematic review and meta-analysis. Fertil Steril 109:698-707. https://doi.org/10.1016/j.fertnstert.2017.11.033

12. LeBlang SD, Hoctor K, Steinberg FL (2010) Leiomyoma shrinkage after MRI-guided focused ultrasound treatment: report of 80 patients. AJR Am J Roentgenol 194:274-280

13. Mindjuk I, Trumm CG, Herzog P, Stahl R, Matzko M (2015) MRI predictors of clinical success in MR-guided focused ultrasound (MRgFUS) treatments of uterine fibroids: results from a single center. Eur Radiol 25:1317-1328. https://doi.org/10.1186/2050-57363-S1-O99

14. Verpalen IM, Anneveldt KJ, Nijholt IM et al (2019) Magnetic resonance-high intensity focused ultrasound (MR-HIFU) therapy of symptomatic uterine fibroids with unrestrictive treatment protocols: a systematic review and meta-analysis. Eur J Radiol 120: 108700. https://doi.org/10.1016/j.ejrad.2019.108700

15. Duc NM, Keserci B (2018) Review of influential clinical factors in reducing the risk of unsuccessful MRI-guided HIFU treatment outcome of uterine fibroids. Diagn Interv Radiol 24:283-291. https:// doi.org/10.5152/dir.2018.18111

16. Fennessy FM, Tempany CM, McDannold NJ et al (2007) Uterine leiomyomas: MR imaging-guided focused ultrasound surgeryresults of different treatment protocols. Radiology 243:885-893. https://doi.org/10.1148/radiol.2433060267

17. Ikink ME, Nijenhuis RJ, Verkooijen HM et al (2014) Volumetric MR-guided high-intensity focused ultrasound versus uterine artery embolisation for treatment of symptomatic uterine fibroids: comparison of symptom improvement and reintervention rates. Eur Radiol 24:2649-2657. https://doi.org/10.1007/s00330-014-3295-6

18. Ikink ME, Voogt MJ, Verkooijen HM et al (2013) Mid-term clinical efficacy of a volumetric magnetic resonance-guided high-intensity focused ultrasound technique for treatment of symptomatic uterine fibroids. Eur Radiol 23:3054-3061. https://doi.org/10.1007/ s00330-013-2915-x

19. Voogt MJ, Trillaud H, Kim YS et al (2012) Volumetric feedback ablation of uterine fibroids using magnetic resonance-guided high intensity focused ultrasound therapy. Eur Radiol 22:411-417. https://doi.org/10.1007/s00330-011-2262-8

20. Ikink ME, van Breugel JMM, Schubert G et al (2015) Volumetric MR-guided high-intensity focused ultrasound with direct skin cooling for the treatment of symptomatic uterine fibroids: proofof-concept study. Biomed Res Int 2015:1-10. https://doi.org/10. 1155/2015/684250

21. Funaki K, Fukunishi H, Funaki T, Sawada K, Kaji Y, Maruo T (2007) Magnetic resonance-guided focused ultrasound surgery for uterine fibroids: relationship between the therapeutic effects and signal intensity of preexisting T2-weighted magnetic resonance images. Am J Obstet Gynecol 196:1-184. https://doi.org/10.1016/j. ajog.2006.08.030
22. Funaki KM, Fukunishi HM, Funaki TP, Kawakami CP (2007) Midterm outcome of magnetic resonance-guided focused ultrasound surgery for uterine myomas: from six to twelve months after volume reduction. J Minim Invasive Gynecol 14:616-621. https://doi. org/10.1016/j.jmig.2007.04.009

23. National Cancer Institute, National Institutes of Health, US Department of Health and Human Services (2017) Common terminology criteria for adverse events (CTCAE). Version 5.0. Available via https://ctep.cancer.gov/protocolDevelopment/electronic applications/docs/CTCAE_v5_Quick_Reference_8.5x11.pdf. Accessed 13 Nov 2019

24. Stewart EA, Gostout B, Rabinovici J, Kim HS, Regan L, Tempany CMC (2007) Sustained relief of leiomyoma symptoms by using focused ultrasound surgery. Obstet Gynecol 110:279-287. https:// doi.org/10.1097/01.AOG.0000275283.39475.f6

25. Yoon SW, Lee C, Cha SH et al (2008) Patient selection guidelines in MR-guided focused ultrasound surgery of uterine fibroids: a pictorial guide to relevant findings in screening pelvic MRI. Eur Radiol 18:2997-3006. https://doi.org/10.1007/s00330-008-1086-7

26. Dobrotwir A, Pun E (2012) Clinical 24 month experience of the first MRgFUS unit for treatment of uterine fibroids in Australia. J Med Imaging Radiat Oncol 56:409-416. https://doi.org/10.1111/j. 1754-9485.2012.02376.x

27. Froeling V, Meckelburg K, Schreiter NF et al (2013) Outcome of uterine artery embolization versus MR-guided high-intensity focused ultrasound treatment for uterine fibroids: long-term results. Eur J Radiol 82:2265-2269. https://doi.org/10.1016/j.ejrad.2013. 08.045

28. Mohr Sasson A, Machtinger R, Mashiach R et al (2018) Longterm outcome of MR-guided focused ultrasound treatment and laparoscopic myomectomy for symptomatic uterine fibroids. J Minim Invasive Gynecol 25:S63. https://doi.org/10.1016/j.jmig. 2018.09.111

29. Gorny KRP, Borah BJP, Brown DLM, Woodrum DAM, Stewart EAM, Hesley GKM (2014) Incidence of additional treatments in women treated with MR-guided focused US for symptomatic uterine fibroids: review of 138 patients with an average follow-up of 2.8 years. J Vasc Interv Radiol 25:1506-1512. https://doi.org/10.1016/ j.jvir.2014.05.012

30. Funaki K, Fukunishi H, Sawada K (2009) Clinical outcomes of magnetic resonance-guided focused ultrasound surgery for uterine myomas: 24-month follow-up. Ultrasound Obstet Gynecol 34:584589. https://doi.org/10.1002/uog.7455

31. Laughlin-Tommaso SM, Barnard EP, AbdElmagied AM et al (2019) FIRSTT study: randomized controlled trial of uterine artery embolization vs focused ultrasound surgery. Am J Obstet Gynecol 220:1-174. https://doi.org/10.1016/j.ajog.2018.10.032

32. Barnard EP, AbdElmagied AM, Laughlin-Tommaso SK et al (2017) Periprocedural outcomes comparing fibroid embolization and focused ultrasound: a randomized controlled trial and comprehensive cohort analysis. Am J Obstet Gynecol 216:500.e1-500.e11

33. Mara M, Maskova J, Fucikova Z, Kuzel D, Belsan T, Sosna O (2008) Midterm clinical and first reproductive results of a randomized controlled trial comparing uterine fibroid embolization and myomectomy. Cardiovasc Intervent Radiol 31:73-85

34. Keltz J, Levie M, Chudnoff SSH (2017) Pregnancy outcomes after direct uterine myoma thermal ablation: review of the literature. J Minim Invasive Gynecol 24:538-545. https://doi.org/10.1016/j. jmig.2017.01.009

35. Okada A, Morita Y, Fukunishi H, Takeichi K, Murakami T (2009) Non-invasive magnetic resonance-guided focused ultrasound treatment of uterine fibroids in a large Japanese population: impact of the learning curve on patient outcome. Ultrasound Obstet Gynecol 34:579-583

36. Kim YS, Lim HK, Rhim H (2016) Magnetic resonance imagingguided high-intensity focused ultrasound ablation of uterine 
fibroids: effect of bowel interposition on procedure feasibility and a unique bowel displacement technique. PLoS One 11:e0155670

37. Keserci B, Duc NM (2018) Magnetic resonance imaging parameters in predicting the treatment outcome of high-intensity focused ultrasound ablation of uterine fibroids with an immediate nonperfused volume ratio of at least 90\%. Acad Radiol 25:12571269. https://doi.org/10.1016/j.acra.2018.01.022

38. Hectors SJCG, Jacobs I, Moonen CTW, Strijkers GJ, Nicolay K (2016) MRI methods for the evaluation of high intensity focused ultrasound tumor treatment: current status and future needs. Magn Reson Med 75:302-317. https://doi.org/10.1002/mrm.25758

39. Jacobs I, Hectors SJCG, Schabel MC, Grüll H, Strijkers GJ, Nicolay K (2015) Cluster analysis of DCE-MRI data identifies regional tracer-kinetic changes after tumor treatment with high intensity focused ultrasound. NMR Biomed 28:1443-1454. https://doi.org/10.1002/nbm.3406

40. Wijlemans JW, Deckers R, van den Bosch MA et al (2013) Evolution of the ablation region after magnetic resonance-guided high-intensity focused ultrasound ablation in a Vx2 tumor model. Invest Radiol 48:381-386. https://doi.org/10.1097/RLI. 0b013e3182820257

41. Younas K, Hadoura E, Majoko F, Bunkheila A (2016) A review of evidence-based management of uterine fibroids. Obstet Gynaecol 18:33-42. https://doi.org/10.1111/tog.12223

Publisher's note Springer Nature remains neutral with regard to jurisdictional claims in published maps and institutional affiliations. 